\title{
SHARPLY TRANSITIVE LINEAR GROUPS OVER ALGEBRAICALLY CLOSED FIELDS
}

\author{
GREGORY CHERLIN, THEO GRUNDHÖFER, \\ ALI NESIN, AND HELMUT VÖLKLEIN
}

(Communicated by Warren J. Wong)

\begin{abstract}
We classify the groups from the title. The result is interpreted in terms of nearfields, and applied to a problem in the model theory of permutation groups.
\end{abstract}

\section{INTRODUCTION}

We prove the following theorem on sharply transitive linear groups.

Theorem. Let $K$ be an algebraically closed field and $G$ a subgroup of $G L_{n}(K)$ which acts sharply transitively on the set of nonzero vectors in $K^{n}$. Then either $n=1$ and $G=K^{*}$, or $n=2$ and the following holds: $G=Z G \cdot G_{0}$ is the product of its center $Z G$ (a group of scalars) with $G_{0}=G \cap \mathrm{SL}_{2}(K)$, where $Z G \cap G_{0}=\{ \pm 1\}$; furthermore, there is a real closed subfield $L$ of $K$ such that $K=L(\sqrt{-1})$, and an L-subalgebra $D$ of the matrix algebra $M_{2}(K)$, isomorphic to the quaternion division algebra over $L$, such that $G_{0} \cong \mathrm{SU}_{2}(K, L)$ is the group of elements of $D$ of norm 1.

We remark that for $n=2$ it would be enough to assume that $K$ is quadratically closed; then the only change is that $L$ is no longer real closed, but Euclidean (i.e., an ordered field whose positive elements are all squares).

The second and fourth author's interest in this theorem came from the theory of nearfields and planes coordinatized by them. In these terms, the theorem amounts to the classification of the nearfields over algebraically closed fields. This can be seen as a counterpart to Zassenhaus' well-known classification of the finite nearfields [Z].

The first and third author's interest in this issue arose from a problem in the model theory of permutation groups. A permutation group $G$ is said to be sharply $n$-transitive on the set $X$ if it acts regularly on the set of ordered $n$-tuples of distinct elements of $X$. The third author has proved that an infinite sharply 3-transitive superstable group is of the form $P S L_{2}(k)$ with $k$

Received by the editors December 22, 1989.

1980 Mathematics Subject Classification (1985 Revision). Primary 03C60, 20H20, 51J20. 
algebraically closed, in its natural action on the projective line [Ne1], and has conjectured that infinite sharply 2-transitive groups of finite U-rank are similarly of standard form, namely: $k_{+} \rtimes k^{\times}$with $k$ an algebraically closed field. This conjecture naturally divides in two parts: that any such group splits as $N \rtimes H$ with $N$ some normal subgroup and with $H$ the stabilizer of a point; and that the split groups are standard. All finite sharply 2-transitive groups are split, and it is possible that this also holds for all infinite ones (with no stability hypothesis). Our theorem yields the following information concerning the split case (Corollary 4, 2 ):

Corollary. Let $G=N \rtimes H$ be a split infinite sharply 2-transitive superstable group of finite U-rank. If the centralizer of $H$ in End $N$ is infinite, then $G$ is of standard form. In particular superstable nearfields of finite U-rank of characteristic 0 are algebraically closed fields.

\section{NeARFIELDS OVER ALGEBRAICALLY CLOSED FIELDS}

The main theorem can be rephrased in the language of nearfields, and applied to the model theoretic problem considered in the introduction. We make those consequences of the theorem explicit here.

A nearfield $(F,+, *)$ is called a Dickson nearfield if there is a division ring $D=(F,+, \cdot)$ and a mapping $\alpha: D^{*} \rightarrow \operatorname{Aut}(D)$ such that $x * y=x \cdot \alpha(x) y$ for all $x, y \in D^{*}$; then the map $\alpha$ satisfies $\alpha(x \cdot \alpha(x) y)=\alpha(x) \alpha(y)$. We say that such a Dickson nearfield is of Kalscheuer type if $D$ is a quaternion division algebra over a real closed field $L$ and $\alpha$ is constant on the norm classes of $D$. Then $\alpha$ is the composition of the norm map $D^{*} \rightarrow L^{+}$(= the group of positive elements of $L)$ and an arbitrary homomorphism $\beta: L^{+} \rightarrow D^{*} / L^{*}(=\operatorname{Aut}(D))$. (Since the image of $\beta$ is abelian, it is actually contained in $K^{*} / L^{*}$ for some 2-dimensional subfield $K$ of $D$ ). As an Abelian group, $L^{+}$is just a vector space over the rationals; hence there is a vast number of such maps $\beta$.

Nearfields of this type were first studied by Kalscheuer [K] in his work on continuous nearfields, where $D$ is the division ring of real quaternions and $\beta$ is continuous, hence of the form $\beta(t)=\exp (i \lambda \log (t)) \bmod L^{*}$ for some real parameter $\lambda$.

Recall that the kernel of a nearfield is the subnearfield (actually a division ring) consisting of the elements $x$ satisfying both $x(y+z)=x y+x z$ and $(y+z) x=y x+z x$ for all $y, z$. Now we can restate our theorem as

Corollary 1. Let $F$ be a nearfield that is of finite dimension $n$ over an algebraically closed field $K$ contained in the kernel of $F$. Then either $n=1$ and $F=K$, or $n=2$ and $F$ is a Dickson nearfield of Kalscheuer type.

An immediate consequence of this is

Corollary 2. Let $F$ be a nearfield that is finite-dimensional over a division ring $H$ contained in the kernel of $F$. If $H$ is a quaternion algebra over some real closed field, then $F=H$. 
We now turn to the model theory of permutation groups. Let $N \rtimes H$ be an infinite sharply 2-transitive group. Then $N$ is abelian and $H$ acts regularly on $N^{\times}$(see e.g. [Ke], 5.11-12). Let $E$ be the additive endomorphism ring of $N$ and view $H$ as a subgroup of the invertible elements of $E$, acting on the right.

Lemma. $C_{E}(H)$ is interpretable in $G$.

Proof. Fix $x \in N^{\times}$. If $f \in C_{E}(H)$ and $f(x)=x^{\prime}$, then for $y \in N^{\times}$we have $y=x^{h}$ for some unique $h \in H$, and $y^{f}=x^{\prime h}$. It is easy to derive an interpretation for $C_{E}(H)$ from this.

Corollary 3. Let $\mathscr{G}=N \rtimes H$ be a split sharply 2-transitive group which is superstable of finite U-rank. If $K=C_{E}(H)$ is infinite then $K$ is an algebraically closed field and $\mathscr{G} \cong K_{+} \rtimes K^{\times}$.

Proof. $K$ is a superstable ring. As $N$ is $H$-irreducible, $K$ is a division ring, hence an algebraically closed field [Ch]. Let $V$ be $N$ with its $K$-structure, and $G=H$. Then $n=\operatorname{dim} V \leq \mathrm{U}$-rk $G$. This restores the notation of the theorem. If $n=1$ we are done. If $n=2$ then $L$ is interpretable in $\mathscr{G}$ and hence is superstable, hence algebraically closed, a contradiction.

Corollary 4. A superstable nearfield of finite U-rank with infinite center is an algebraically closed field.

This applies in particular if $F$ is of characteristic zero.

It is reasonable to conjecture that infinite superstable sharply 2-transitive groups are all of standard form, and in particular that superstable nearfields are commutative. (It is known that superstable fields are either finite or algebraically closed.)

One extreme possibility we have not been able to eliminate is the following: $G=A \rtimes H, A$ is abelian with no proper infinite definable subgroups, $|Z H|=$ $2, H / Z H$ a bad group of Morley rank 3. In particular $H / Z H$ would be a simple linear group with no involution; it is not known whether such groups exist at all.

The model theory of nearfields has been investigated by Felgner [Fe], who looked at the pseudofinite case, and also by Schulz [S].

\section{THE PROOF OF THE MAIN THEOREM}

The hypotheses and notation of the main theorem are in force throughout this section. Let $K^{*}$ denote the multiplicative group of $K$. As $K$ is algebraically closed, all $g \in G$ have nontrivial eigenvectors. By the regularity hypothesis, elements of $G$ commute if and only if they have a common eigenvector, and are conjugate if and only if they have a common eigenvalue. We refer to these criteria as the commutation and conjugacy criteria below. Clearly $G$ acts irreducibly on $V:=K^{n}$; hence by Schur's Lemma, the center $Z G$ acts as a group of scalars.

We will now proceed to prove the theorem in a series of lemmas, using in- 
duction on $n$. The case $n=1$ is trivial, so assume $n>1$. For $g \in G$ let $C(g)$ denote the centralizer of $g$ in $G$.

Lemma 1. If $g \in G-Z G$ and $V_{\alpha} \neq(0)$ is the $\alpha$-eigenspace of $g$ in $V$ (for some $\alpha \in K)$, then $V_{\alpha}$ has dimension 1, and the map that sends each $h \in C(g)$ to the eigenvalue of $h$ on $V_{\alpha}$ is an isomorphism from $C(g)$ to $K^{*}$.

Proof. $C(g)$ acts on $V_{\alpha}$, and we claim first that it acts regularly on the nonzero vectors of $V_{\alpha}$. If $v, w \neq 0$ are in $V_{\alpha}$ and $h$ is the (unique) element of $G$ with $h \cdot v=w$, then $[g, h] v=v$ and thus $h \in C(g)$. The regularity of $C(g)$ on $V_{\alpha} \backslash\{0\}$ follows. Now if the dimension $d$ of $V_{\alpha}$ is 1 , the claim follows. So assume $d>1$. Then it follows by induction that $d=2$ and $C(g)$ has the structure specified in the conclusion of the theorem. In particular $G$ contains a noncentral element $g^{\prime}$ of order 4, which has $\pm \sqrt{-1}$ as its eigenvalues. Since the characteristic of $K$ is not 2 and $g^{\prime}$ is of order $4, g^{\prime}$ is diagonalizable with two distinct eigenvalues, and it must have an eigenspace $V^{\prime}$ of dimension $\geq 2$ since $n>2$. Again it follows that $\operatorname{dim} V^{\prime}=2$ and $C\left(g^{\prime}\right)$ has the structure specified in the conclusion of the theorem. Thus $C\left(g^{\prime}\right)$ has both a central element (namely, $g^{\prime}$ ) and a noncentral element of order 4. By the conjugacy criterion they are conjugate in $C\left(g^{\prime}\right)$ (since $C\left(g^{\prime}\right)$ acts regularly on $V^{\prime}$ ), which is a contradiction.

Lemma 2. Let $g, g^{\prime} \in G-Z G$.

(a) The elements $g, g^{\prime}$ commute if and only if they have the same set of eigenspaces.

(b) We have either $C(g)=C\left(g^{\prime}\right)$ or $C(g) \cap C\left(g^{\prime}\right)=Z G$.

(c) If $N(g)$ denotes the normalizer of $C(g)$ in $G$, then $N(g) / C(g)$ acts regularly on the set of eigenspaces of $g$.

(d) $C(g)$ and $C\left(g^{\prime}\right)$ are conjugate.

Proof. (a) follows from the commutation criterion and the fact that the eigenspaces are 1-dimensional. Claim (b) follows from (a).

For the transitivity in (c), let $v, w$ be eigenvectors for $g$ and let $h$ be the element of $G$ with $h v=w$. Then $g$ and $g^{h}$ have $v$ as an eigenvector; hence $g \in C(g) \cap C\left(g^{h}\right)$ (by the commutation criterion); thus $C(g)=C\left(g^{h}\right)$ (by (b)), and so $h \in N(g)$. This shows the transitivity. On the other hand if $h V_{\alpha}=V_{\alpha}$ with $V_{\alpha}$ the $\alpha$-eigenspace of $g$, then $h \in C(g)$ (by the commutation criterion), which shows the regularity.

For (d), after a suitable conjugation we can assume $g, g^{\prime}$ share a common eigenvector, and apply $(a, b)$.

Lemma 3. (a) $n=2$, and $\operatorname{char}(K) \neq 2$.

(b) Every $g \in G-Z G$ has 2 distinct eigenvalues, and $[N(g): C(g)]=2$.

Proof. Let $g \in G-Z G$. By Lemma 2(c) the number $m$ of distinct eigenvalues of $g$ is $[N(g): C(g)]$, which is independent of $g$ by Lemma 2(d). Suppose $m=1$, and write $n=q^{r} e$ where $q=\operatorname{char}(K)$ (or $q=1$, if $\operatorname{char}(K)=0$ ), and $e \not \equiv 0(\bmod q)$. Then any element $f$ of the commutator subgroup $G^{\prime}$ of 
$G$ has as its only eigenvalue an $e$ th root of unity (since $\operatorname{det}(f)=1$ ); hence $f^{e}$ has eigenvalue 1 and so $f^{e}=1$. Since $X^{e}-1$ is a separable polynomial, $f$ is diagonalizable, hence is a scalar $\lambda \cdot \mathrm{id}_{V}$, with $\lambda$ an $e$ th root of unity. So $G^{\prime}$ is finite and central in $G$. Hence the commutator yields a bi-multiplicative map $G \times G \rightarrow G^{\prime}$, and this map must be trivial since $G$ is divisible and $G^{\prime}$ is finite. Thus $G$ is abelian, and so $G=Z G$ a group of scalars, which is absurd.

Thus $m>1$, and so there is $h \in N(g)$ whose image in $N(g) / C(g)$ has prime order $p$. Then Lemma 2(b) shows

$$
C(g) \cap C(h)=Z G .
$$

Since $h^{p} \in C(g)$ it follows that

$$
h^{p} \in Z G
$$

In particular since $h$ has more than one eigenvalue, the characteristic of $K$ is not $p$. The element $h$ induces an automorphism $\sigma$ of order $p$ of $C(g)$. Let $U_{p}$ be the group of elements of $C(g)$ of $p$-power order. Since $C(g) \cong K^{*}$, $U_{p}$ is a Prüfer group, and its automorphism group is isomorphic to $\mathbb{Z}_{p}^{\times}$, the invertible $p$-adic integers.

Now we show $p=2$. If $p>2$ there is no element of order $p$ in $\mathbb{Z}_{p}^{\times}$(cf. [Am]), so $U_{p} \subseteq C(g) \cap C(h)=Z G$. Now $Z G$ is the group of fixed points of the automorphism $\sigma$ of prime order $p$ acting on a divisible Abelian group, whose $p$-torsion lies in $Z G$; hence $Z G$ is $p$-divisible (in additive notation, if $p x \in$ $Z G$ and $p y=x$ then $\sigma y=y+z$ with $p^{2} z=0$; hence $y=\sigma^{p} y=y+p z$ and $p z=0, \sigma x=x)$. As $h^{p} \in Z G$ and $Z G$ is $p$-divisible, we can take $h^{p}=1$. Now if $v$ is an eigenvector for $g$, then $h$ fixes the vector $v+h v+\cdots+h^{p-1} v$, which is nontrivial by Lemma 2 (c). This is a contradiction, which shows that $p=2$.

Now $h^{2}$ is a scalar (by (2)); hence $h$ has at most two distinct eigenvalues. Thus $m \leq 2$. Since $m>1$, we have $m=2$. Furthermore since $\operatorname{char}(K) \neq 2$, $h$ is diagonalizable, with 1-dimensional eigenspaces (Lemma 1), so $n=2$. This proves (a).

As $m=2$, (b) follows from Lemma 2(c).

Lemma 4. Let $G_{0}=G \cap \operatorname{SL}(2, K)$. Then $G=Z G \cdot G_{0}$ with $Z G \cap G_{0}=\{ \pm 1\}$. Proof. Clearly $-1 \in G$ (since an element of $G$ of order 2 can only have one eigenvalue); hence $Z G \cap G_{0}=\{ \pm 1\}$. Let $g \in G-Z G$. As $[N(g): C(g)]=2$ and $C(g)$ is divisible abelian with a unique involution, if $h \in N(g)-C(g)$ then $h$ induces an automorphism of order 2 on $C(g)$ and $g=g_{+} g_{-}$where $h$ centralizes $g_{+}$and inverts $g_{-}$. Then $g_{+} \in Z G$ and $g_{-}$is conjugate to its inverse, and has two distinct eigenvalues. Since these eigenvalues cannot be the pair $\{1,-1\}$ they must be a pair $\left\{\alpha, \alpha^{-1}\right\}$ and thus $g_{-} \in G_{0}$.

To identify the group $\bar{G}_{0}:=G_{0} /\langle-1\rangle$, we will use the geometry of involutions of $\bar{G}_{0}$, inspired by Bachmann [Ba]. 
Notation. For $g \in G_{0}$ let $C_{g}=C_{G_{0}}(g)$ and $N_{g}=N_{G_{0}}\left(C_{g}\right)$. Let $\bar{G}_{0}=$ $G_{0} /\langle-1\rangle$.

Lemma 5. Let $g \in G_{0}-\{ \pm 1\}$. Then:

(1) $C_{g}$ is abelian and 2-divisible.

(2) $\left[N_{g}: C_{g}\right]=2$.

(3) $N_{g}-C_{g}$ consists of elements of order 4, each of which acts on $C_{g}$ by inversion.

(4) All elements of order 4 in $G$ lie in $G_{0}$, and ar conjugate in $G_{0}$.

(5) The involutions of $\bar{G}_{0}$ are exactly the images in $\bar{G}_{0}$ of elements of order 4 in $G_{0}$.

Proof. (5) is clear, and we know $C_{g}$ is abelian.

If $h \in N(g)-C(g)$ then $h \equiv h^{\prime} \bmod Z G$ for some $h^{\prime} \in N_{g}-C_{g}$ (by Lemma 4). Thus $\left[N_{g}: C_{g}\right] \geq 2$. Also $N_{g}-C_{g} \subseteq N(g)-C(g)$; hence $\left[N_{g}: C_{g}\right]=2$. By the proof of Lemma 4, each $h \in N_{g}-C_{g}$ acts on $C_{g}$ by inversion; in particular, $h^{2} \in C_{g}$ must be an involution, and hence $h$ is of order 4. This proves (2) and (3).

By the conjugacy criterion one sees that all elements of $G$ of order 4 are conjugate, and (4) follows.

It remains to show that $C_{g}$ is 2-divisible. By Lemma $4, C_{g} /( \pm 1) \cong K^{\times} / Z G$ is 2-divisible, and by (4) it follows that $C_{g}$ is also 2-divisible.

Lemma 6. For any two distinct involutions of $\bar{G}_{0}$ there is another involution in $\bar{G}_{0}$ distinct from the two and centralizing both.

Proof. Let $i, j \in G_{0}$ be elements of order 4 representing distinct involutions $\bar{l}, \bar{\jmath}$ of $\bar{G}_{0}$. Let $g=i j \notin\{ \pm 1\}$. Then $C(g)$ contains a unique cyclic subgroup $\langle k\rangle$ of order 4 , and this $k$ is in $G_{0}$. As $i, j$ invert $g$, they normalize $C(g)$, hence either invert or centralize $k$. In either case $\bar{l}, \bar{\jmath}$ centralize $\bar{k}$. If $\bar{l}, \bar{\jmath}$ do not commute, then clearly they are distinct from $\bar{k}$; if they do commute, then $\bar{k}=\bar{g}=\overline{\imath \jmath}$, which again implies $\bar{k} \neq \bar{\imath}, \bar{k} \neq \bar{\jmath}$.

Lemma 7. $G_{0}$ equals its own commutator subgroup $G_{0}^{\prime}$.

Proof. By Lemma 5.3, we have for each $g \in G_{0}$ that $g^{-1}=g^{j}$ for some $j \in G_{0}$. Thus $g^{-2}=[g, j] \in G_{0}^{\prime}$. So $G_{0}^{\prime}$ contains all squares of $G_{0}$; but by Lemma 5.1, each element of $G_{0}$ is a square.

Now let $A$ be the 3-dimensional $\mathrm{K}$-vector space of all trace 0 matrices in $\mathrm{M}_{2}(K)$. The determinant yields a nondegenerate quadratic form $Q$ on $A$. The group $G_{0}$ acts on $A$ by conjugation, leaving $Q$ invariant. This gives an embedding of $\bar{G}_{0}=G_{0} /\langle-1\rangle$ into the special orthogonal group $\mathrm{SO}_{3}(Q)$, and via this embedding we will regard $\bar{G}_{0}$ as a subgroup of $\mathrm{SO}_{3}(Q)$. Let $I$ (resp., $I_{0}$ ) be the set of involutions in $\mathrm{SO}_{3}(Q)$ (resp., in $\bar{G}_{0}$ ). 
Lemma 8. (a) Each involution $\nu \in I$ has a 1-dimensional eigenspace $A^{+}(\nu)$ and a 2-dimensional eigenspace $A^{-}(\nu)$, corresponding to the eigenvalue +1 and -1 , respectively. These eigenspaces are nondegenerate (relative to $Q$ ), and for each nondegenerate 1-space (resp., 2-space) $X$ in $A$ there is a unique $\nu \in I$ with $X=A^{+}(\nu)$ (resp., $X=A^{-}(\nu)$ ).

(b) For distinct $\nu, \nu_{1}, \nu_{2} \in I$, the following are equivalent:

(i) $\nu$ centralizes $\nu_{1}$ and $\nu_{2}$.

(ii) $A^{+}(\nu)=A^{-}\left(\nu_{1}\right) \cap A^{-}\left(\nu_{2}\right)$.

(iii) $A^{-}(\nu)=A^{+}\left(\nu_{1}\right)+A^{+}\left(\nu_{2}\right)$.

Proof. An elementary exercise in Linear Algebra (all contained in [Ba]).

Now let $\mathscr{P}$ (resp., $\mathscr{L}$ ) denote the set of all $A^{+}(\nu)$ (resp., $A^{-}(\nu)$ ) for $\nu \in I_{0}$. Then $\mathscr{P}$ (resp., $\mathscr{L}$ ) is a set of points (resp., lines) of the projective plane $\pi_{A}$ associated with $A$, and we are going to show that $(\mathscr{P}, \mathscr{L})$ is a subplane of $\pi_{A}$. Take $\nu_{1} \neq \nu_{2}$ in $I_{0}$. By Lemma 6 there is $\nu \in I_{0}$ distinct from $\nu_{1}, \nu_{2}$, and centralizing $\nu_{1}, \nu_{2}$. Hence by Lemma 8(b), the lines of $\mathscr{L}$ (resp., the points of $\mathscr{P}$ ) associated to $\nu_{1}$ and $\nu_{2}$ intersect in the point of $\mathscr{P}$ (resp., are joined by the line of $\mathscr{L}$ ) associated to $\nu$. Clearly, $(\mathscr{P}, \mathscr{L})$ is not contained in a triangle; hence it is a subplane of $\pi_{A}$.

Choose points $K b_{1}, \ldots, K b_{4}$ in $\mathscr{P}$ forming a (nondegenerate) quadrangle. Then $b_{1}, b_{2}, b_{3}$ form a basis of $A$, and we may assume $b_{4}=b_{1}+b_{2}+b_{3}$. This gives homogeneous coordinates on $\pi_{A}$ such that the above points have coordinates $(1,0,0),(0,1,0),(0,0,1)$ and $(1,1,1)$, respectively. Then it follows from the usual geometric interpretation of addition and multiplication that the elements $t \in K$ for which the point $(t, 0,1)$ lies in $\mathscr{P}$ form a subfield $L$ of $K$. Furthermore, $\mathscr{P}$ consists exactly of those points in $\pi_{A}$ with some coordinate triple in $L^{3}$. In other words, if $\pi_{B}$ denotes the projective plane associated to the $L$-vector space $B=L b_{1}+L b_{2}+L b_{3}$, then the canonical image of $\pi_{B}$ in $\pi_{A}$ equals the subplane $(\mathscr{P}, \mathscr{L})$.

Lemma 9. $\bar{G}_{0}$ leaves $B$ invariant.

Proof. Viewing PGL $(A)$ as a collineation group of $\pi_{A}$, we first note that the identity is the only element of $\operatorname{PGL}(A)$ acting trivially on the subplane $\pi_{B}$ (namely, such an element would in particular fix four points forming a quadrangle). Hence the subgroup $\Phi$ of $\operatorname{PGL}(A)$ fixing $\pi_{B}$ is isomorphic to a collineation group of $\pi_{B}$ containing PGL $(B)$. But the stabilizer in $\Phi$ of any quadrangle in $\pi_{B}$ is trivial; hence $\Phi$ coincides with $\operatorname{PGL}(B)$ (embedded naturally into PGL $(A))$. Thus the inverse image of $\Phi$ in GL $(A)$ is $\Psi:=K^{*} \cdot \mathrm{GL}(B)$.

Now $\bar{G}_{0}$ is contained in $\Psi$, hence also in the commutator subgroup of $\Psi$ (by Lemma 7). Thus $\bar{G}_{0} \subseteq \mathrm{SL}(B)$, which means that $\bar{G}_{0}$ leaves $B$ invariant.

Every 1-dimensional subspace of $B$ is of the form $A^{+}(\nu) \cap B$ for some $\nu \in I_{0}$. Since all $\nu \in I_{0}$ are conjugate under $\bar{G}_{0}$ (Lemma 5.4, 5.5) it follows 
that $\bar{G}_{0}$ acts transitively on the 1 -spaces in $B$. Thus we may assume det $(b) \in L$ for all $b \in B$ (replacing $B$ by $\alpha B$ for some $\alpha \in K$, if necessary); further, if $b \neq 0$ then $\operatorname{det}(b) \neq 0$ (since $b$ lies in some space $A^{+}(\nu)$, which is $Q$ anisotropic). Thus det gives an anisotropic quadratic form on $B$, invariant under $\bar{G}_{0}$. Viewing $B$ as an anisotropic quadratic space via det, we get $\bar{G}_{0} \subseteq$ $\mathrm{SO}_{3}(B)$.

Lemma 10. (a) $[K: L]=2$; hence $L$ is a real closed field and $\operatorname{char}(K)=0$.

(b) The eigenvalues $x$ of each $g \in G_{0}$ have norm $N_{K / L}(x)=1$.

Proof. Let $x, x^{-1}$ be the eigenvalues of some $g \in G_{0}$. By Lemma 5.1 we have $x=y^{2}$ where $y, y^{-1}$ are the eigenvalues of some $h \in G_{0}$. Then $y^{2}, y^{-2}, 1$ are the eigenvalues of its image $\bar{h} \in \bar{G}_{0}$ acting on $A$.

Assuming (a) momentarily, we derive (b). For $x, y, g, h$ as above, since $\left.\bar{h}\right|_{B} \in \mathrm{SO}_{3}(B)$, it follows from (a) that $1=\mathrm{N}_{K / L}\left(y^{2}\right)=\mathrm{N}_{K / L}(x)$, proving (b).

Thus it only remains to prove (a). With $x, y, g, h$ as above, since $\bar{h}$ fixes $B$, the trace $\operatorname{tr}(\bar{h})=y^{2}+y^{-2}+1$ lies in $L$. Now $\operatorname{tr}(g)=x+x^{-1}=y^{2}+y^{-2} \in L$, so we get

For all $g \in G_{0}$ we have $\operatorname{tr}(g) \in L$.

Since $x$ is an eigenvalue of $\bar{h}$ on $A$, if $x \in L$ then $\bar{h}$ has an eigenvector $v$ in $B$ with eigenvalue $x$, and $\operatorname{det}\left(v^{h}\right)=x^{2} \operatorname{det}(v)$; since det is anisotropic on $B$, we find $x= \pm 1$. This shows:

If $x \neq \pm 1$ is an eigenvalue of some $g \in G_{0}$, then $x \notin L$.

By Burnside's theorem [Ja, p. 213], $G_{0}$ contains a basis $g_{1}, \ldots, g_{4}$ of the matrix algebra $\mathrm{M}_{2}(K)$. Since the trace form on $\mathrm{M}_{2}(K)$ is nondegenerate, the map $\phi: \mathrm{M}_{2}(K) \rightarrow K^{4}$ sending any $m \in \mathrm{M}_{2}(K)$ to the 4-tuple $\left(\operatorname{tr}\left(m g_{1}\right), \ldots, \operatorname{tr}\left(m g_{4}\right)\right)$ is a linear isomorphism. Additionally, $\phi\left(G_{0}\right) \subseteq L^{4}$ by (1). Hence $G_{0} \subseteq L m_{1}+\cdots+L m_{4}$ for certain $m_{1}, \ldots, m_{4} \in \mathrm{M}_{2}(K)$. Thus for $w \neq 0$ in $K^{2}$ we have $G_{0} \cdot w \subseteq L m_{1}(w)+\cdots+L m_{4}(w)$. So the $L$-vector space $W$ spanned by $G_{0} \cdot w$ has dimension $\leq 4$ over $L$.

Clearly, the set $k$ of all $t \in K$ with $t W \subseteq W$ is a subfield of $K$, with $[k: L] \leq 4$. Since $k w(\subseteq K w)$ cannot exhaust all of $W$, we even get $[k: L] \leq 2$. If $x$ is an eigenvalue of some $g \in G_{0}$ then $x w \in G_{0} \cdot w$; hence $x W \subseteq W$ and so $x \in k$. Now (2) shows that $k \neq L$. Hence $[k: L]=2$. Thus $W$ has dimension 2 over $k$.

Choose a basis $w_{1}, w_{2}$ of $W$ over $k$. Then this is also a basis of $K^{2}$ (over $K)$. Now any line in $K^{2}$ through 0 whose slope in $w_{1}, w_{2}$-coordinates does not lie in $k$ intersects $W$ trivially. Hence such a line cannot exist (since $G_{0}$ acts transitively on the lines in $K^{2}$ through 0 , and leaves $W$ invariant). This proves $k=K$, hence $[K: L]=[k: L]=2$. The rest of the claim follows. 
Let $i \in K$ with $i^{2}=-1$. Since $\left.\operatorname{det}\right|_{B}$ is an anisotropic quadratic form over the real closed field $L$, its nonzero values are all positive or all negative (in the unique order on $L$ ). Replacing $B$ by $i B$ if necessary, we may thus assume $\operatorname{det}(b)>0$ for all $b \neq 0$ in $B$. Any $g \in M_{2}(K)$ can be written uniquely as $g=\mu \cdot 1+b_{1}+i b_{2}$ with $\mu \in K, b_{1}, b_{2} \in B$. For $g \in G_{0}$, since $g$ centralizes itself and normalizes $B$, we get $b_{1}^{g}=b_{1}$ and $b_{2}^{g}=b_{2}$. If $g \neq \pm 1$, it follows that $b_{1}$ and $b_{2}$ are linearly dependent over $L$ (since each nonidentity element of $\mathrm{SO}_{3}(B)$ has a 1-dimensional fixed space in $B$ ). Thus every $g \in G_{0}$ is of the form $g=\mu \cdot 1+\lambda b$ for some $b \in B, \lambda, \mu \in K$. Now $\mu=\operatorname{tr}(g) / 2$, and $\operatorname{tr}(g) \in L$ (by (1) from the proof of Lemma 10). Furthermore, $1=\operatorname{det}(g)=\mu^{2}+\lambda^{2} \operatorname{det}(b)$; hence $\lambda^{2} \in L$. Now we will show that $\lambda^{2} \geq 0$, so that $\lambda \in L$, and we may conclude that:

Lemma 11. $G_{0}$ is contained in $D:=L \cdot 1+B$.

Proof. To show that $\lambda^{2} \geq 0$ it suffices to show that $\mu^{2} \leq 1$ (since $\operatorname{det}(b) \geq 0$ by the choice of $B$ above). But the eigenvalues $x, x^{-1}$ of $g$ have norm 1 (by Lemma 10) and satisfy $\mu=\left(x+x^{-1}\right) / 2$. Thus $|\mu|=\left|x+x^{-1}\right| / 2 \leq$ $\left(|x|+\left|x^{-1}\right|\right) / 2=1$ (where $|$.$| is the usual absolute value, i.e., the square root$ of $\mathrm{N}_{K / L}$ ). Thus also $\mu^{2} \leq 1$.

Since $G_{0}$ contains 4 linearly independent elements, it follows that the $L$ span of $G_{0}$ equals $D$. Thus $D$ is a subalgebra of $\mathrm{M}_{2}(K)$. Any $d \neq 0$ in $D$ is of the form $d=\mu \cdot 1+b$ with $\mu \in L, b \in B$ not both zero; hence $\operatorname{det}(d)=\mu^{2}+\operatorname{det}(b)>0$. Thus $D$ is a (4-dimensional) division algebra over $L$, hence is the quaternion (division) algebra over $L$. Furthermore $G_{0}$ is contained in the group $\mathrm{SU}_{2}(K, L)$ of quaternions of norm 1, and from the transitive action of $G_{0}$ on the 1-spaces in $K^{2}$ it follows that $G_{0}$ contains all elements of $\mathrm{SU}_{2}(K, L)$ of order 4 . These elements generate the group, and hence $G_{0}=\mathrm{SU}_{2}(K, L)$.

\section{REFERENCES}

[Am] Y. Amice, Les nombres p-adiques, Presses Univ. France, Paris, 1975.

[Ba] F. Bachmann, Aufbau der Geometrie aus dem Spiegelungsbegriff, Die Grundlehren der mathematischen Wissenschaften, Springer-Verlag, Berlin-Göttingen-Heidelberg, 1959.

[Ch] G. Cherlin, Superstable division rings, Logic Colloquium 1977, North-Holland, Amsterdam, 1978, pp. 99-111.

[Fe] U. Felgner, Pseudofinite nearfields, Near-rings and Near-fields (Betsch, ed.), North-Holland, Amsterdam, 1987.

[Ja] N. Jacobson, Basic algebra II, 2nd ed., W. H. Freeman and Company, 1989.

[K] F. Kalscheuer, Die Bestimmung aller stetigen Fastkörper über dem Körper der reellen Zahlen als Grundkörper, Abh. Math. Sem. Hamb. Univ. 13 (1940), 413-435.

[Ke] W. Kerby, On infinite sharply transitive multiply transitive groups, Hamburger Mathematische Einzelschriften, 1974.

[Ne1] A. Nesin, On sharply n-transitive superstable groups, J. Pure Appl. Algebra (to appear).

[Ne2] A. Nesin, Nonsolvable groups of Morley rank 3, J. Algebra 124 (1989), 199-218. 
[S] K. U. Schulz, Undecidability of the theory of finite nearfields, Resultate Math. 14 (1988), 340-348.

[Z] H. Zassenhaus, Über endliche Fastkörper, Abh. Math. Sem. Hamb. Univ. 11 (1936), 187-220.

Department of Mathematics, Rutgers University, Hill Center, New Brunswick, New JERSEY 08903

Mathematical Institute auf Der MoRgenstelle 10, Universität TÜBingen, D-7400 TÜBINGen, Federal RePUblic OF Germany

Department of Mathematics, University of California at Irvine, Irvine, California 92717

Department of Mathematics, University of Florida, Gainesville, Florida 32611 